\title{
Evaluating Precise Quantity of Decommissioning Waste by Cutting Virtual 3D Models of Large Equipment
}

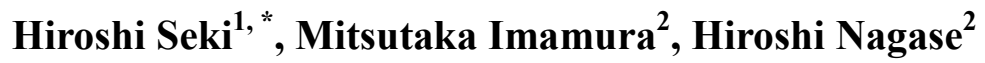 \\ ${ }^{1}$ Research \& Development Group, Hitachi, Ltd., Hitachi-shi, Japan \\ ${ }^{2}$ Nuclear Engineering and Product Division, Hitachi-GE Nuclear Energy, Ltd., Hitachi-shi, Japan
}

Email address:

hiroshi.seki.mf@hitachi.com(H. Seki)

*Corresponding author

\section{To cite this article:}

Hiroshi Seki, Mitsutaka Imamura, Hiroshi Nagase. Evaluating Precise Quantity of Decommissioning Waste by Cutting Virtual 3D Models of Large Equipment. Nuclear Science. Vol. 5, No. 3, 2020, pp. 36-43. doi: 10.11648/j.ns.20200503.12

Received: October 1, 2020; Accepted: October 22, 2020; Published: October 30, 2020

\begin{abstract}
Equipment and piping components contaminated by radioactive materials and/or containing low-level irradiated waste must be cut, segmented, and packed into waste containers. Workers need to avoid overexposure to radiation in dismantling environments, and the number of waste containers for the pieces of equipment and piping components needs to be minimized. Thus, we developed an automatic planning method for virtually cutting 3D equipment with limitations on container size, radioactivity, weight, and dose rate. Cutting sequence data was used to formulate different cutting-work procedures, generate cut objects, and calculate the exposure during disassembling work. By calculating the required cutting length and dose-rate distribution in working environments for various cutting sequences of large equipment, the developed system is expected to aid in the planning of decommissioning. To utilize systems engineering in conjunction with elemental technologies, the following problems need to be solved; both weight and volume of the waste need to be controlled so that radioactive waste for decommissioning nuclear power plants is traceable. Identifying segmented equipment from a 3D model is key to calculating the number of volumetric segmented fragments and required number of containers. To evaluate exposure and amount of waste, we developed an automatic planning method for virtually cutting 3D equipment objects given constraints. Cutting sequence data was used to formulate different cutting workflows, generate cut objects, and calculate the exposure dose from disassembling work.
\end{abstract}

Keywords: Decommissioning, 3D-CAD, Dose-Rate Visualization, Waste-Quantity Estimation, Virtual Cutting Plane

\section{Introduction}

Decommissioning projects are expected to increase for nuclear power plants (NPPs) that have been in operation for many years. And also, the decommissioning projects for NPPs need to be carried out safely, efficiently, and economically. Decommissioning radioactive waste is $3.6 \%$ of the total waste in a commercial boiling water nuclear power plant [1]. The total cost of dismantling processes, including cutting, processing, and removing waste materials in a decommissioning project, is $73 \%$ of the total amount of decommissioning cost [2]. At the same time, the radiation exposure dose for dismantling work must be within the speculated value provided in safety regulations. Therefore, decommissioning projects must be planned optimally with consideration for work duration and exposure dose. In the past thirty years, several decommissioning projects have been completed, and related project-management systems have been developed. A database for dismantling and evaluating workload, radiation exposure dose, waste weight, as well as schedules of the dismantling processes were managed by the Code System for Management of Decommissioning (COSMARD) [3] for the Japan Demonstration Power Reactor (JPDR). Since then, research institutes in Japan and Norway have developed a Decommissioning Engineering Support System (DEXUS) for identifying an appropriate dismantling plan for a decommissioning project [4]. Virtual reality (VR) methods have been applied in conjunction with the VRDOSE software tool to simulate and plan dismantling work in an environment contaminated by radioactivity [5-7]. The distribution dose rate can be computed with numerical 
calculation codes that take into account source types and locations, the geometry of the environment, and the materials' characteristics [8-10]. A cutting process simulation has been developed and tested for the waste from equipment and piping components [11].

Hitachi has been developing decommissioning engineering support systems on the basis of 3D computer aided design (CAD) models [12, 13]. Decommissioning engineering essentially involves evaluating a residual radioactive inventory, planning for decontamination, dismantling, remotely controlling dismantling machines, managing waste processing, and measuring radiation. Decommissioning projects should proceed on the basis of these procedures. To integrate them, the 3D geometric shapes of a decommissioning plant, functional system data, and data on residual radioactivity accumulated during plant operation should be stored into a database and shared by decommissioning support systems. Basic functions for evaluating the quantity of decommissioning waste and the worker's accumulated dose by cutting virtual 3D models of large equipment have been developed to provide the above systems with essential data.

In this paper, the problem and the objective of this research are described in Chapter 2. Chapter 3 describes the method for automatically generating cutting objects using 3D models of large equipment. The simulation method for various possible cutting procedures is then described in Chapter 4, and the simulation results and discussion are presented in Chapter 5. Finally, the conclusion is given in Chapter 6.

\section{Problem Statement and Objectives of the Research}

In Japan, funds for decommissioning are allocated while nuclear plants are still in operation. It is necessary to estimate the actual cost of decommissioning after power generation is permanently shut down to avoid running out of funds. The technologies required for decommissioning can be divided into elemental technologies and systems engineering [14]. Decommissioning projects need to integrate both in order to be properly carried out. A database containing geometric shapes, function (system) data for the decommissioned nuclear plant, and contamination from radioactive materials accumulated during operation is needed to link the elemental technologies and make appropriate use of systems engineering.

To utilize systems engineering in conjunction with elemental technologies, the following problems need to be solved:

1. Both weight and volume of the waste need to be controlled so that radioactive waste for decommissioning nuclear power plants is traceable.

2. Identifying segmented equipment from a 3D model is key to calculating the number of volumetric segmented fragments and required number of containers.

3. There are tradeoffs between avoiding overexposure to radiation and minimizing number of waste containers with multiple scenarios for segmenting equipment and piping component.

4. Different cutting workflows should be formulated for generating cut objects and calculating the exposure dose for disassembling work, particularly for cutting sequence data.

\section{Method for Automatic Segmentation Based on Equipment 3D Model}

\subsection{System Configuration for Integrated Cutting and Dismantling Simulation}

We developed an automatic method for generating 3D cut objects from equipment models with internal structures for planning the dismantling of equipment in the decommissioning phase of NPPs (Figure 1). Segmented 3D objects were stored with cutting conditions obtained from equipment $3 \mathrm{D}$ models. The equipment 3D models with the distribution of radioactivity were used for calculating the environmental dose rate and the interim dose rate during dismantling work. The number of man-hours and exposure dose for the dismantling work were also calculated after simulating the dismantling sequences with segmented 3D objects, typical dismantling sequences, and interim dose rates at tentative cutting states. Finally, waste container models were generated with segmented 3D objects and used to automatically calculate the trajectory for removing the waste containers from their original locations, putting them in a provisional storage place, and then moving them outside the plant.

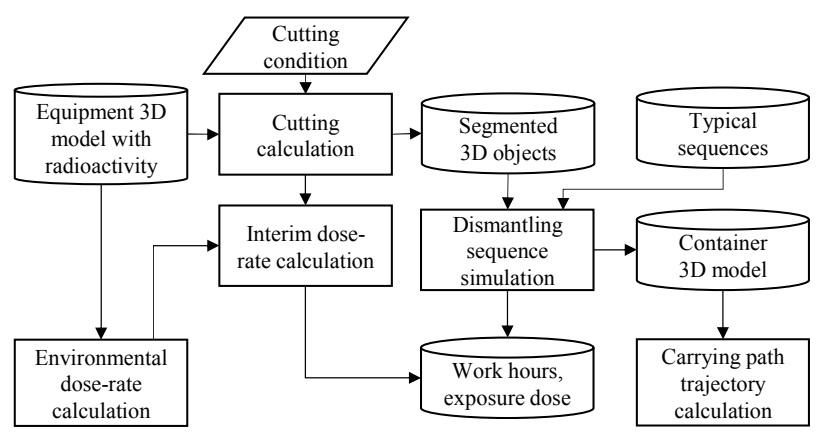

Figure 1. System configuration for integrated cutting and dismantling simulation.

\subsection{Automatic Segmentation of 3D Model for Large Equipment}

In this development, 3D models for large equipment such as heat exchangers, tanks, and pumps were automatically cut using the following procedure (Figure 2):

(1) Prepare the original equipment model with internal structures and define virtual cutting planes.

(2) Superimpose the equipment model onto the horizontal and vertical cutting planes and generate cut objects from both sides of the cutting planes. Then, uniquely identify the cut objects by the IDs of the objects. 
(3) Convert the cut objects to a general 3D CAD format file that can easily be used for other tools (such as viewers), dismantling, and carrying out simulations.

(4) As for the geometric shapes of heat exchangers (i.e., cylinders), adjust the distances between the cutting planes and the cylindrical axis and the number of divisions in the circumference directions from the user interfaces.

Data on the cut objects were stored in a database, and the number of cut fragments (C0001, C0002, etc. in the case of equipment number AA-HX-13-1 for the 13-1th heat exchanger (HX) in the system AA) was calculated. The above method was used to generate fragments under various constraints during dismantling and to generate a management hierarchy of fragments for traceability.

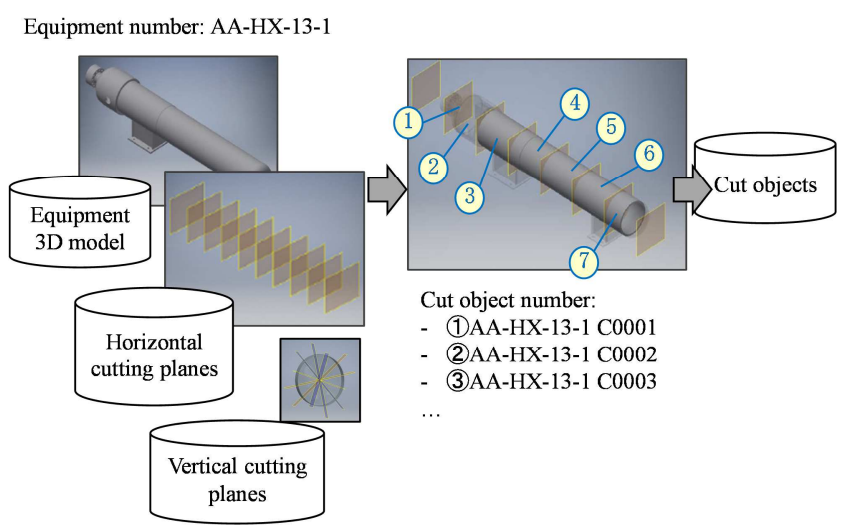

Figure 2. Detailed 3D model of equipment cutting based on virtual planes.

\subsection{Optimization of Cutting Length}

Figure 3 shows a flowchart for generating geometric shapes for cut fragments under waste container constraints. The geometric shapes were generated in the following three steps:

(1) Step 1: Setting virtual cutting planes

Since most of the large equipment resembled cylindrical or cubic shapes, multiple virtual cutting planes were placed perpendicular to the axis in the longest direction of the equipment shape, and the intervals (lengths) between adjacent virtual cutting planes were equal. Two types of interval lengths were set so as to correspond to the manual and automatic adjustment of virtual planes. One type of interval length was fixed groups with pre-defined cutting lengths. The other was variable interval groups depending on the limitations on radioactivity concentration, container size, and weight.

(2) Step 2: Setting constraints for cutting dimensions

The maximum interval lengths were calculated to satisfy waste container limitations for the automatic calculation method. The equal interval lengths were calculated from the user's designations, such as the number of cutting planes and the length of intervals.

(3) Step 3: Adjusting cutting lengths

The distance intervals were determined by repeatedly calculating the cutting lengths of fragment objects in automatic mode until the requirements for the waste container, such as internal volume, maximum radioactivity concentration, maximum surface-dose rate, and maximum weight storable in the selected waste container, were satisfied.

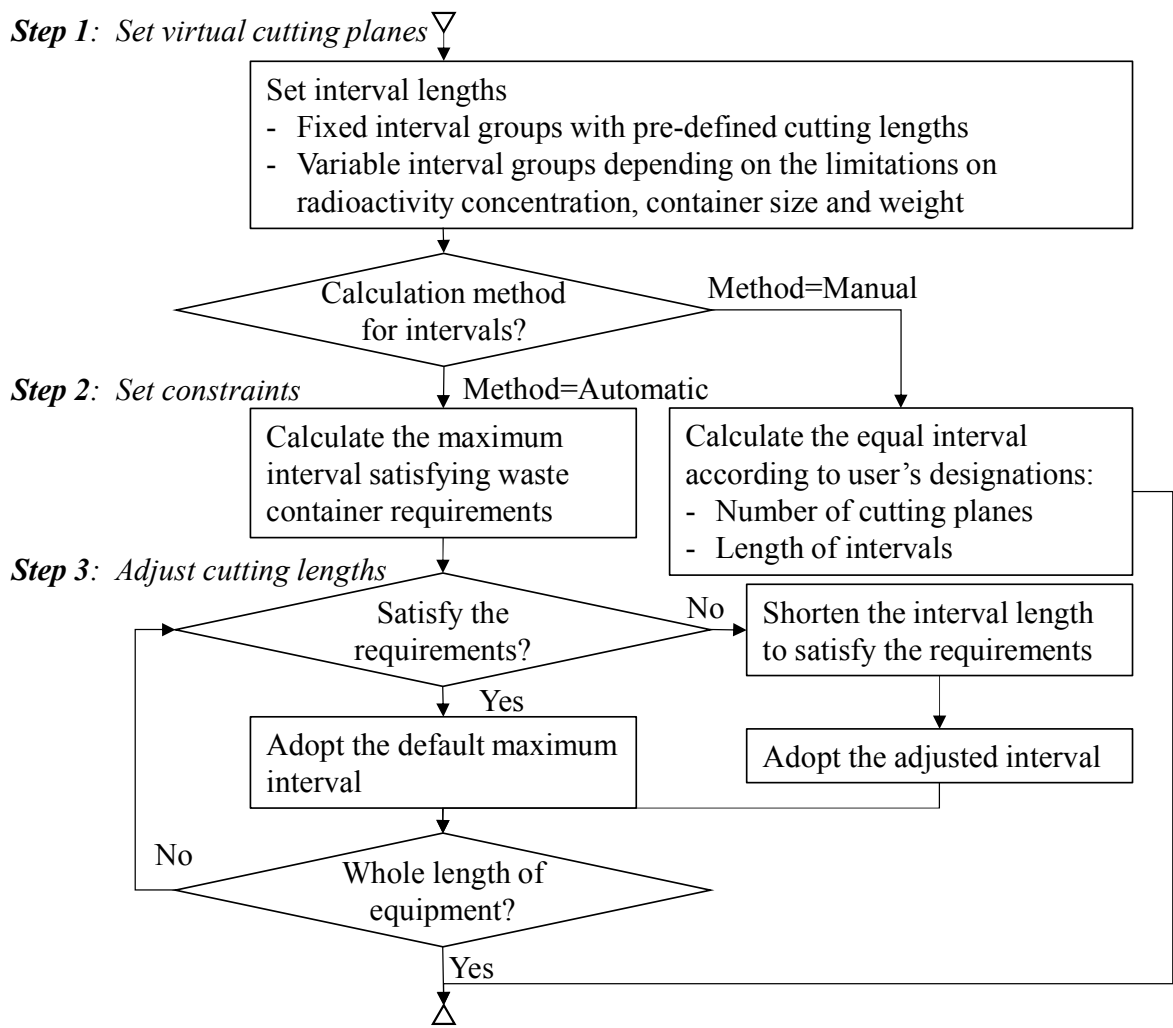

Figure 3. Flowchart for arranging virtual cutting planes. 
The cutting-interval length and the number of divisions in the axial and circumferential directions to satisfy the constraints on waste containers were calculated for the cylindrical equipment. In this calculation, the dimensions of the cut fragments were adjusted according to the internal volume of the specific waste container. The concentration of radioactivity, maximum surface dose rate, and total weight of cut waste fragments were then evaluated to determine whether those values were under the stipulated limits when the cut fragments were packed into the waste container. If the radioactive concentration and/or the total weight were not within the limits, the cutting interval was shortened and the values were evaluated again until they were within the limits. The number of cutting divisions for the circumferential direction was selected from 180, 90, 60, 45, and 30 degrees to generate equal objects by cutting.

The cutting lengths were fine-tuned in reference to the lines made by the virtual cutting planes perpendicular to the axis for the longest dimension. The cutting interval was also set by manually designating the number of cutting planes and the size of intervals between the virtual cutting planes.

The maximum dose rate was calculated using the Particle and Heavy Ion Transport Code System (PHITS) [15]. If the dose rate exceeds the limit of the value stipulated for a specific waste container, the system sounds a warning to indicate that the condition of the packed waste fragments is not within the surface-dose-rate limit

\subsection{Management of Hierarchy for Segmented Components}

A bill of materials (BOM) is changed regularly during disassembling processes. The hierarchy and nodes of the BOM were managed in accordance with the following rules:

(1) Unique IDs must be assigned to each node on the generated fragments according to the cutting and disassembling schedule.

(2) The origins of the generated fragments must be represented as parent-child relationships in the hierarchy.

The changing hierarchy of the cutting objects is shown in Figure 4. Assembly001 depicts a cross-sectional view of a heat exchanger, which is composed of Part001 (the outer body) and Subassembly001 (a bundle of small-bore tubes). These parts were cut along the dotted lines. The equipment labelled "Part" was treated as an object with no child components before cutting, whereas the equipment labelled "Subassembly" was treated as an object with child components. In this example, Subassembly001 comprises of Part002, Part003, Part004, etc. Information concerning the parts and components in the BOM before cutting was provided, and a BOM database was prepared so that the system could manage such information.

Sub assembly 001

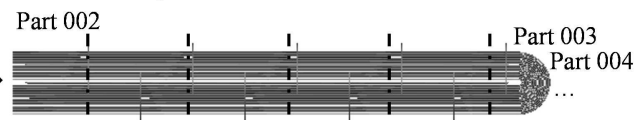

Bulk 001 iBulk 002i Bulk 003iBulk 004iBulk 005і Bulk 006 $<$ Before Cutting $>$

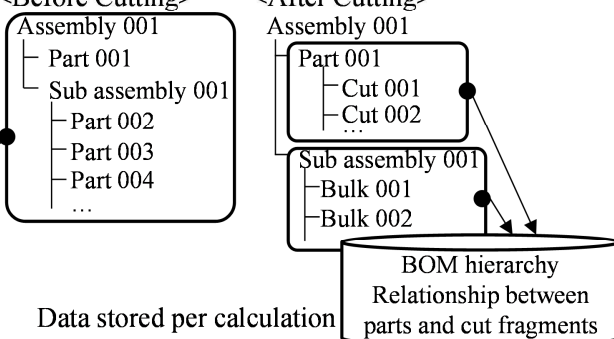

Figure 4. Bill of material (BOM) hierarchy for cutting objects.

After cutting, Part001 was cut into fragments Cut001, Cut002,..., and Cut006. Likewise, Subassembly001 was cut into Bulk001, Bulk002,.., and Bulk006. "Bulk" refers to a bundle of cut fragments, such as tubes, and the cut tubes in the bulk could not be identified uniquely because of the efficiency of handling data. Within this process, the BOM hierarchy was used to manage the information on the cut object fragments and the origins of the parent parts. The relationships between parts and cut fragments were stored in the database every time object cutting was calculated. The cut fragment inherited the attributes of the original parts, and the concentration of radioactivity and weights were allocated according to the volume ratio between the original part and the fragment.

\section{Cutting Procedure for Simulation}

\subsection{Changing Dose-Rate Depending on Cutting Procedure}

To cut large equipment such as heat exchangers, multiple cutting sequences need to be considered while taking into account exposure dose and duration of work to estimate decommissioning costs (Figure 5). For this purpose, the following three cases were studied.

A heat exchanger has internal structures containing a group of small-bore tubes, which are contaminated with radioactive materials such as Co-60 with the fluid from a nuclear reactor core during plant operation. If the tubes are extracted first, 
the remaining body of the heat exchanger is minimally contaminated and the radiation exposure dose rate can be suppressed. Then the body of the heat exchanger can be cut in vertically and horizontally. The extracted tubes should be cut separately, so it takes double the cutting time, however the exposure dose can be decreased with moderate shields. These cutting sequences are referred to as Procedure A.
The heat exchanger with the internal tubes can be cut horizontally first and then vertically, as in Procedure B. The heat exchanger can also be cut vertically first, and then horizontally (Procedure C). Both Procedures B and C take less time than $\mathrm{A}$; however, the exposure dose may increase because the shields at the cutting situation cannot be set appropriately in front of the cutting objects.

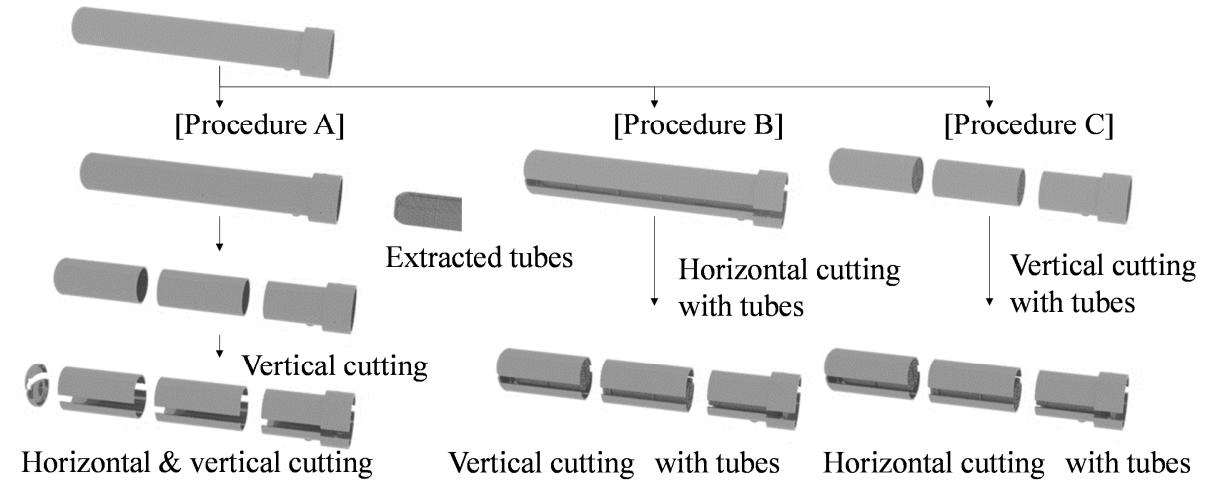

Figure 5. Multiple cutting sequences for heat exchanger with internal tubes.

The exposure from bare radioactive sources in extracted tubes occurs while disassembling cut fragments. The dose-rate distribution drastically changes when the equipment is cut by tools. However, calculating the dose rate at each cutting is impractical and time consuming. The dose rate is based on the allocation of the radioactive sources in the equipment material. Although the radioactive sources are decreased linearly by removing the cut objects from the installed locations, the Monte Carlo calculation using PHITS in small intervals takes a considerably long time. Thus, it was necessary for us to calculate dose-rate more efficiently in cutting work situations.

\subsection{Timing of Movement for Cut Fragment Objects}

In addition to cutting, removal tasks are also performed during dismantling. Radioactive fragments are moved from their original locations to a provisional storage space. As a result, the dose rate in the work environment varies during the disassembling processes.

To calculate the changing dose-rate in the work environment, typical work states were selected to reduce calculation times. The attribute values were set to indicate the type of work as "Dose" in the parameters for multiple cutting sequences, as shown in Table 1. In each state of "Dose", the dose-rate distribution at the corresponding cutting, namely "procedure name" and "sequence number," was calculated. The dose-rate distributions at the other sequence numbers were not calculated for the types of work such as "Cut". If the complex behaviour of workers are simulated in detail without this kind of table, many parameters and dose rate calculations need to be prepared for calculating the exposure dose. With the help of the uniform table, the exposure doses that depend on several cutting sequences can be calculated more efficiently.

Table 1. Parameters for multiple cutting sequences.

\begin{tabular}{|c|c|c|c|c|c|c|c|c|c|c|}
\hline \multirow[b]{2}{*}{$\begin{array}{l}\text { Procedure } \\
\text { name }\end{array}$} & \multirow[b]{2}{*}{ Sequence } & \multirow[b]{2}{*}{$\begin{array}{l}\text { Group } \\
\text { name }\end{array}$} & \multirow[b]{2}{*}{$\begin{array}{l}\text { Cutting } \\
\text { method }\end{array}$} & \multirow[b]{2}{*}{$\begin{array}{l}\text { Type of } \\
\text { work }\end{array}$} & \multicolumn{4}{|c|}{ Placement coefficient } & \multicolumn{2}{|c|}{ Surface coefficient } \\
\hline & & & & & $\begin{array}{l}\text { Place the } \\
\text { object "as } \\
\text { is", } p_{1}\end{array}$ & $\begin{array}{l}\text { Transporting } \\
\text { to another } \\
\text { place, } p_{2}\end{array}$ & $\begin{array}{l}\text { Transporting } \\
\text { adjacent } \\
\text { parts, } p_{3} \\
\end{array}$ & $\begin{array}{l}\text { Stacking } \\
\text { objects, } \\
p_{4} \\
\end{array}$ & $\begin{array}{l}\text { Caps on cut } \\
\text { surfaces, } s_{1}\end{array}$ & $\begin{array}{l}\text { Insulations } \\
\text { on cut } \\
\text { surfaces, } s_{2} \\
\end{array}$ \\
\hline Procedure A & 1 & G001 & K001 & Cut & 1 & 3 & 3 & 3 & 1 & 1 \\
\hline Procedure A & 2 & G002 & K002 & Cut & 1 & 1 & 1 & 1 & 1 & 1 \\
\hline Procedure A & 3 & Null & Null & Dose & 3.52 & 1 & 1 & 1 & 1 & 0.92 \\
\hline Procedure A & $\ldots$ & $\ldots$ & $\ldots$ & $\ldots$ & $\ldots$ & $\ldots$ & $\ldots$ & $\ldots$ & $\ldots$ & $\ldots$ \\
\hline Procedure B & 2 & G002 & K001 & Cut & 1 & 1 & 1 & 1 & 1 & 1 \\
\hline Procedure B & $\ldots$ & $\ldots$ & $\ldots$ & $\ldots$ & $\ldots$ & $\ldots$ & $\ldots$ & $\ldots$ & $\ldots$ & $\ldots$ \\
\hline Procedure C & 1 & G001 & K001 & Cut & 1 & 1 & 1 & 1 & 1 & 1 \\
\hline Procedure C & 2 & Null & Null & Dose & 3.52 & 1 & 1 & 1 & 1 & 0.92 \\
\hline Procedure C & $\ldots$ & $\ldots$ & $\ldots$ & $\ldots$ & $\ldots$ & $\ldots$ & $\ldots$ & $\ldots$ & $\ldots$ & $\ldots$ \\
\hline
\end{tabular}

The same table was used when taking into account both the timing of the movement for cutting fragment objects and the dependencies of cutting sequences on different paths. The column labelled "group name" was used for the cutting activities with a group of parallel virtual cutting planes. The method corresponding to the tools used for disassembling was labelled as "cutting method".

Different cutting work flows (as shown in Figure 5) were 
formulated with this sequence data and used to generate cut objects and calculate the exposure dose from the disassembling work.

To calculate the factors affected by the movement of cut objects, placement coefficients were set for treating various situations of placing the cut fragments, such as placing the objects on provisional places as is $\left(p_{1}\right)$, transporting objects to another place $\left(p_{2}\right)$, moving adjacent parts $\left(p_{3}\right)$, and stacking cut objects in temporary areas $\left(p_{4}\right)$. As a safety measure, the effects of the exposure dose rates from the radioactive sources inside the cut objects were treated using the coefficients for insulation effects by placing caps on cut surfaces $\left(s_{1}\right)$ and insulation on cut surfaces $\left(s_{2}\right)$. These coefficients were used to calculate the maximum dose rate in working environments $D_{\max }$ as

$$
D_{\max }=S_{\max } \times \prod_{i=1}^{4} p_{i} \times \prod_{j=1}^{2} S_{j}
$$

Where $S_{\max }$ is the maximum dose rate when there are no shields. The exposure dose can be calculated by the product of $D_{\max }$ and work hours in the equation (1).

Multiple cutting sequences are shown in Figure 5. For instance, after a bundle of small-bore tubes is extracted, the outer body can be cut horizontally and vertically in Procedure A. In Procedure B, the tubes can be cut horizontally and cut vertically again, and in Procedure $\mathrm{C}$, the tubes are vertically and horizontally cut. The exposure dose from each disassembling work sequence depends on these cutting sequences, since radioactive materials exist inside the heat exchangers.

\section{Results and Discussion}

\subsection{Surface Dose Rate on Waste Containers}

Figure 6 shows a visualization of the calculated dose-rate distribution surrounding waste fragments from a large piece of equipment. Gamma radiation penetrated the surface of the waste container, and the maximum surface dose-rate spot was calculated by comparing the dose-rate values on the surface. Then, the system automatically checked whether the packed fragments and the waste container satisfied the regulations.

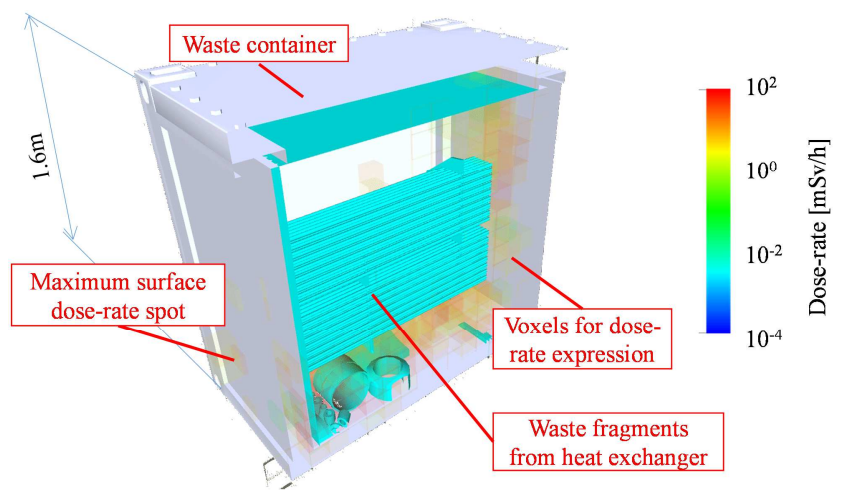

Figure 6. Visualization of a waste container with packed waste fragments and dose-rate distribution.

In this case, the radioactivity of Co-60 for the waste fragments was $9 \times 10^{8} \mathrm{~Bq} / \mathrm{t}$. This concentration of radioactivity was categorized as very low-level waste. After 100 years, the Co-60 radioactivity level will decrease to about $10^{-6}$ since the half-life of Co-60 is 5.3 years. Then the above radioactivity will become $9 \times 10^{2} \mathrm{~Bq} / \mathrm{t}$ and the radioactive waste will be regarded as cleared materials because it is less than $0.1 \mathrm{~Bq} / \mathrm{g}$ $\left(=10^{5} \mathrm{~Bq} / \mathrm{t}\right)$. This reduction of radioactivity is significant because it is not necessary for us to treat the cleared materials as radioactive waste. To do so, the identification and allocation of radioactivity would be useful for tracking the radioactivity of each fragment.

\subsection{Dependency of Accumulated Dose on Type of Cutting Procedures}

The exposure dose rate depends on the cutting procedure and time. If the cutting lengths are shorter, the exposure dose tends to become higher than that of longer lengths because the cutting time is increased, as shown in Figure 7. In Procedure A, the tubes of the heat exchanger are removed, and then the body and the tubes of the heat exchanger are cut separately. Therefore, the exposure dose of dismantling the heat exchanger with Procedure A becomes higher than that of Procedures B and $\mathrm{C}$. This is due to the placement coefficients $p_{i}$, even though the surface coefficients $s_{j}$ are sufficiently low. This evaluation of exposure provides basic information on workers' exposure dose during dismantling tasks for equipment and piping components contaminated with radioactive materials.

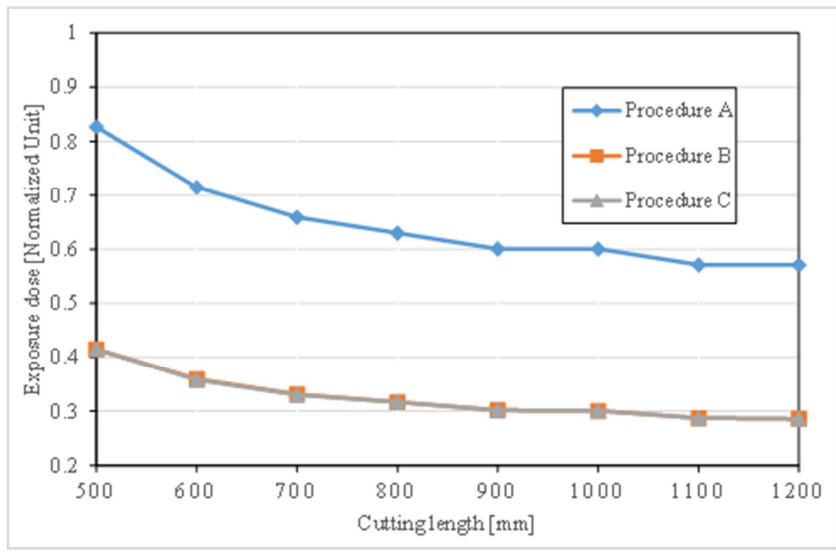

Figure 7. Accumulated dose dependency on cutting procedures.

\subsection{Calculated Results of Waste Quantities and Required Number of Waste Containers}

The relationship between the number of fragments and cutting length was investigated with the developed system for the integrated cutting and dismantling simulation. As shown in Figure 8 , the number of fragments decreases when the cutting length increases. The procedure shown in Figure 5 affected the number of fragments, and if the internal small-bore tubes were cut together with the external body of the heat exchanger as in Procedure C, the number of fragments are almost same among Procedures A, B and $\mathrm{C}$. Due to the complexity of the geometry and calculation errors, the calculated waste volume is slightly different between Procedure $\mathrm{C}$ and Procedure A and B. This result indicates that similar geometric shapes should be cut within their group to 
reduce the number of objects comprising the waste fragments. While the number of waste fragments decreased when the cutting length was increased, the number of required waste containers increased to 10 to 14 . This increase in containers was due to the complexity of the geometric shapes. The decreasing number of waste fragments was flattened between $900 \mathrm{~mm}$ to $1000 \mathrm{~mm}$. This flattened range was affected by the remainders of the tubes and the body of the heat exchanger less than $1000 \mathrm{~mm}$ which were cut $900 \mathrm{~mm}$ or $1000 \mathrm{~mm}$ each.

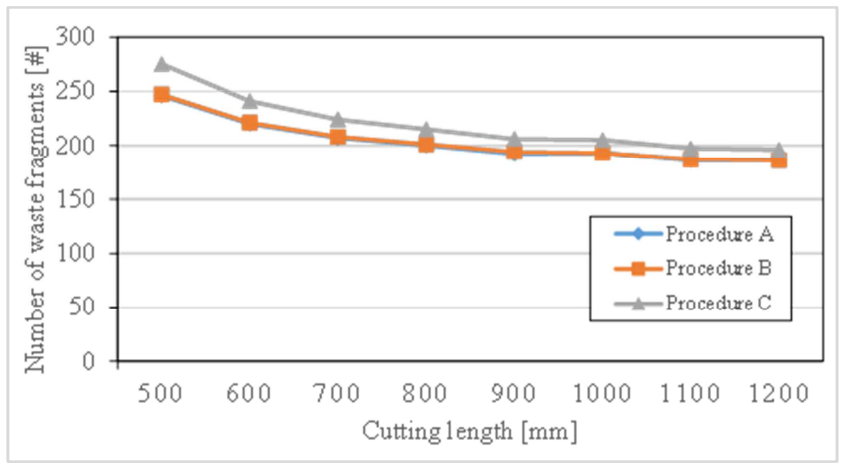

Figure 8. Relationship between number of fragments and cutting length.

\section{Conclusion}

The objective of this research was to minimize the risk of overexposure to radiation and reduce the number of waste containers needed for cutting equipment and piping components into fragments. To evaluate exposure and amount of waste, we developed an automatic planning method for virtually cutting 3D equipment objects given constraints on container size, radioactivity, weight, and dose-rate. Cutting sequence data was used to formulate different cutting work flows, generate cut objects, and calculate the exposure dose from disassembling work.

Based on the calculated dose-rate distribution surrounding waste fragments from a large piece of equipment, the maximum surface dose-rate spot was calculated by comparing the dose rate values on the surface. Then, the system automatically checked whether the packed fragments and the waste container satisfied the regulations.

And then the evaluation of exposure shows basic information on workers' exposure dose during dismantling tasks for equipment and piping components contaminated with radioactive materials. As a result of evaluation for waste quantities, the number of fragments decreases when the cutting length increases were estimated precisely based on virtually cutting 3D equipment with limitations on waste container size, radioactivity, weight, and dose rate.

\section{References}

[1] Schmittem M. (2016). Nuclear decommissioning in Japan: Opportunities for European companies, EU-Japan Centre for Industrial Cooperation, https://www.eu-japan.eu/sites/default/files/publications/docs/20 16-03-nuclear-decommissioning-japan-schmittem-min_0.pdf.
[2] Grossi A. et al. (2013). Cost estimation for decommissioning of research reactors, INAC 2013 ISBN 978-85-99141-05-2, https://inis.iaea.org/collection/NCLCollectionStore/ Public/46 /021/46021420.pdf.

[3] Yanagihara, S. (1993) COSMARD: The Code System for Management of JPDR Decommissioning, Journal of Nuclear Science and Technology, 30 (9): 890-899.

[4] Iguchi, Y., Kanehira, Y., Tachibana, M. and Johnsen T. (2004). Development of decommissioning engineering support system (DEXUS) of the Fugen Nuclear Power Plant, Journal of Nuclear Science and Technology, 41 (3): 367-375.

[5] Rindahl, G. and Mark, K. (2008). Innovative and adaptive technologies in decommissioning of nuclear facilities; VRDose and emerging 3D software solutions to support decommissioning activities, IAEA-TECDOC- 1602 147-158.

[6] Szőke, I., Louka, M., Bryntesen, T., Bratteli, J., Edvardsen, S., RøEitrheim, K. and Bodor, K. (2014). Real-time 3D radiation risk assessment supporting simulation of work in nuclear environments, Journal of Radiological Protection 34 (2): 389 416.

[7] Szőke, I., Louka, M., Bryntesen, T., Edvardsen, S. and Bratteli, J. (2015). Comprehensive support for nuclear decommissioning based on 3D simulation and advanced user interface technologies, Journal of Nuclear Science and Technology, 52 (3): 371-387.

[8] Ohga, Y., Fukuda, M., Shibata, K., Kawakami, T. and Matsuzaki, T. (2005). A system for the calculation of radiation field for maintenance support in nuclear power plants Radiation Protection Dosimetry, 116 (1-4): 592-596.

[9] Paviot, T., Mouton, C., and Lamouri, S. (2013). Long term control of $3 \mathrm{D}$ engineering data for nuclear power plants, Proceedings of the 18th International Conference on $3 \mathrm{D} \mathrm{Web}$ Technology, ACM 205.

[10] Lee, J., Kim, G., Kim, I., Hyun, D., Jeong, K., Choi, B., and Moon, J. (2016). Establishment of the framework to visualize the space dose rates on the dismantling simulation system based on a digital manufacturing platform, Annals of Nuclear Energy 95: 161-167.

[11] Kim, I., Choi, B., Hyun, D., Moon, J., Lee, J., Jeong, K. and Kang, S. (2016). A framework for a flexible cutting-process simulation of a nuclear facility decommission, Annals of Nuclear Energy 97: 204-207.

[12] Nonaka, Y., Yamamoto, E., Oya, K., Enomoto, A. and Seki, H. (2016). Development of IT-driven power plant engineering work support systems, Hitachi Review 65 (4): 963-968.

[13] Seki, H., Imamura, M., Kitahara, T., Enomoto, A. (2017). Estimation of decommissioning quantity based on 3D intelligent model, Proceedings of the 45th Annual Waste Management Conference International Conference (WM2017) 17075 .

[14] Ishigure, K. (2003). Decommissioning strategies and programme developments of Japan Proceedings of the SFEN Conference. Decommissioning challenges: An Industrial Reality?

https://inis.iaea.org/search/searchsinglerecord.aspx?recordsFor $=$ SingleRecord $\& \mathrm{RN}=35089854$. 
[15] Sato, T., Niita, K., Matsuda, N., Hashimoto, S., Iwamoto, Y., Noda, S., Ogawa, T., Iwase, H., Nakashima, H., Fukahori, T., Okumura, K., Kai, T., Chiba, S., Furuta, T. and Sihver, L. (2013). Particle and heavy ion transport code system PHITS, Version 2.52, Journal of Nuclear Science and Technology, 50 (9). 913-923. 